\title{
Preparation, Characterization and In-Vitro Release Study of Flurbiprofen Loaded Stealth Liposomes
}

\author{
M.YASMIN BEGUM ${ }^{1 *}$ K. ABBULU² and M. SUDHAKAR ${ }^{3}$ \\ ${ }^{1}$ Department of Pharmaceutics, ${ }^{1}$ Department of Biotechnology, \\ ${ }^{3}$ Malla Reddy College of Pharmacy, Maisammaguda, Secunderabad-500014, India \\ ${ }^{2}$ Malla Reddy Institute of Pharmaceutical Sciences, Maisammaguda, \\ Secunderabad.-500014, India \\ yaminimp47@gmail.com
}

Received 6 May 2012 / Accepted 17 May 2012

\begin{abstract}
The objective of the study was to prepare flurbiprofen loaded stealth liposome formulations by optimizing the various process and formulation related variables. Various liposomal batches were prepared by thin film hydration technique and they were characterized for drug encapsulation \%, chemical interaction of the drug and excipients, vesicle size, drug release profile in vitro and stability using specified methods. The best flurbiprofen loaded liposome was composed of DSPC/Cholesterol group of 4:1 mole ratio and the best stealth liposome was composed of DSPC/CH/ PE 18:0|18:0-PEG 2000 (PE-PEG) group of 4:1:0.2 mole ratios. Stealth liposome was found to have greater stability, higher drug encapsulation $\%$ and lower drug release in vitro compared to others. The drug encapsulation efficiency of stealth liposomes was $68 \%$ and it could retain $70.9 \%$ of the drug even after $24 \mathrm{~h}$. FTIR study indicated that there is no significant chemical interaction between the components. SEM photograph confirmed that vesicles were homogenous and spherical in shape. Stability studies showed that the vesicles were stable in $-20{ }^{\circ} \mathrm{C}$ and refrigerated temperature $\left(4{ }^{\circ} \mathrm{C}\right)$ for one month without significant differences in drug entrapment. The present study has given us knowledge also that liposomes with low amount of cholesterol are better candidate for flurbiprofen liposomes.
\end{abstract}

Keywords: Stealth liposomes, Stability, In vitro release, FTIR, SEM analysis

\section{Introduction}

The German bacteriologist Paul Ehrlich first proposed the expression "magic bullet” which is a sort of drug delivery system that would target only diseased cell, because directing drugs to particular cells continued to be almost a great challenge ${ }^{1}$. Drugs that are the most potent are also the most toxic, because often less than $1 \%$ of the injected drug reaches its target while rest damages healthy cells ${ }^{2}$. The difference between the therapeutic dose and a toxic dose of the most potent drugs is very narrow. One of the main goals of any treatment is to increase the therapeutic index of the drug while minimizing its side effects. The ideal solution would be to target the drug only to the cells or tissues affected by the disease. Selected carriers such as liposomes, niosomes, microspheres, nanospheres, erythrocytes, polymeric and reverse micelles were studied, but by far the most widely studied approach 
makes use of liposomes. Their attraction presents in their composition, which makes them biodegradable and biocompatible ${ }^{3}$. Liposomes consist of an aqueous core enveloped by one or more bilayers of natural or synthetic phospholipids ${ }^{3}$. These are the carriers which are suitable for encapsulation of drugs with different lipophilicities such as strongly lipophilic drugs, strongly hydrophilic drugs and drugs with intermediate $\log \mathrm{P}^{3}$. Liposomes can protect the encapsulated drug or drugs and can reach the target organ or tissue by passive targeting ${ }^{3}$. But it was found that conventional liposomes suffer with two major drawbacks as sustained as well as targeted release system for drugs in vivo. First one is its attraction towards reticuloendothelial system which will cause removal of drug from blood stream as well as results adverse effects on the host defense system ${ }^{4}$ and decrease availability of entrapped drug to the others tissues. The next is recognition of conventional liposomes by reticuloendothelial system leads to non-linear pharmacokinetics for the carrier which makes difficulty of calculating amount of entrapped drug required to attain therapeutic dose $\mathrm{e}^{5-8}$. In addition conventional liposome formulations containing saturated phospholipids and cholesterol are more prone to the influence of plasma proteins and other biological fluids in vivo leads to rapid removal of drug contents ${ }^{9-11}$. To avoid the above mentioned difficulties especially to avoid the MPS uptake of the vesicles it is necessary to have previous administration of empty liposomes. Moreover small uni-lamellar vesicles have the drawback of low aqueous entrapment volume; the use of charged liposomes could be toxic. Thus, mechanical or electrostatic stabilization can not improve the long circulation of liposomes in biological systems ${ }^{2}$. Further attempts to alter the biodistribution of liposomes resulted the generation of new liposomal formulations called as stealth liposomes, have considerably reduced MPS uptake, retain in circulation for long period of time ${ }^{12,13}$ with dose independent pharmacokinetics $^{8}$ and have reduced susceptibility to protein induced leakage ${ }^{14,15}$.

Flurbiprofen that is selected here as the model for hydrophobic drug, is the first candidate of coxibs, a specific cyclooxygenase- 2 inhibitor, a NSAID family that is frequently used for treating pain and inflammation of arthritis ${ }^{16,17}$. Abdominal discomfort and other gastro intestinal side effects are the most frequently reported side effects of flurbiprofen upon oral administration ${ }^{18}$. Flurbiprofen is highly protein bound ${ }^{17}$ and has large volume of distribution. It has high $\log \mathrm{P}$ value of 4.16; short elimination half-life of 3.95 necessitates the drug to be administrated frequently ${ }^{19}$. In such case, the area under the plasma drug concentration versus time curve increases upon increasing dose of drug administration ${ }^{20}$. In order to avoid the above said side effects as well as draw backs associated with flurbiprofen, it was chosen as the drug model for our present study. In the present study we report development of stealth liposomes by studying various process as well as formulation related factors such as drug-lipid ratio, effect of cholesterol, effect of alkyl chain length of lipids which may affect the size, shape and encapsulation efficiency of liposomes. Molecular interactions between flurbiprofen, cholesterol and phospholipids were studied by Fourier transform, infrared (FTIR) spectroscopy. In vitro release rate studies were carried out on specially designed in vitro model.

\section{Experimental}

Flurbiprofen was kindly donated by FDH limited, Mumbai. Soy phosphatidyl choline (SPC), dipalmitoyl phosphatidyl choline (DPPC), distearoyl phosphatidyl choline (DSPC), PE 18:0/18:0-PEG 2000 (PE-PEG) were kindly gifted by lipoid, Germany. High purity cholesterol and sigma membrane (12000 MW cut off) were purchased from Sigma Aldrich, Hyderabad. Potassium dihydrogen phosphate, disodium hydrogen phosphate, sodium chloride, chloroform, methanol and ethanol were purchased from commercial source. Deionized water was used throughout the experiment. 


\section{Preparation of flurbiprofen loaded conventional and stealth liposomes}

Multi lamellar vesicles (MLVs) containing flurbiprofen was prepared by thin film hydration technique ${ }^{21,22}$. Drug and phospholipid with or without cholesterol were dissolved in $10 \mathrm{~mL}$ solvent system of chloroform and methanol mixture $(2: 1, \mathrm{v} / \mathrm{v})$ in a $250 \mathrm{~mL}$ round bottom flask. The organic solvent system was removed by using rotary evaporator (HS 3001 NS) under reduced pressure to obtain a thin film on the wall of the flask. During the process, the conditions such as speed $(150 \mathrm{rpm})$ and temperature $45^{\circ} \pm 2{ }^{\circ} \mathrm{C}$ for conventional liposomes and $55^{\circ} \pm 2^{\circ} \mathrm{C}$ for liposomes with lipids such as DPPC, DSPC and stealth liposomes were maintained constant. The flask was removed and left overnight in a desiccator under reduced pressure to remove the solvent residuals completely. Then the lipid film was hydrated using phosphate buffer saline $\mathrm{pH} 7.4$ at $60 \pm 2{ }^{\circ} \mathrm{C}$. The resultant suspension was vortexed for about $2 \mathrm{~min}$ and allowed to stand for 2-3 h in order to allow complete swelling of the lipid film. Then the suspension was sonicated using probe sonicator for about $5 \mathrm{~min}$ and extruded through polycarbonate membrane of $0.2 \mu \mathrm{m}$ pore size. In the same manner prescribed above flurbiprofen loaded stealth liposomes were prepared by using DSPC, PE 18:0/18:0-PEG and cholesterol. These formulations were used on the same day for further studies.

\section{Fourier transform, infrared (FTIR) study}

All the excipients such as SPC, DPPC, DSPC, PE 18:0/18:0-PEG, cholesterol, pure drug flurbiprofen individually, physical mixture of drug and excipients were mixed separately with infrared (IR) grade $\mathrm{KBr}$ in the ratio of 1:100 and corresponding pellets were prepared by applying $15000 \mathrm{lb}$ of pressure in a hydraulic press. The pellets were scanned in an inert atmosphere over a wave number range of $4000-400 \mathrm{~cm}^{-1}$ in Magna IR 750 series II (Nicolet, USA) FTIR instrument.

\section{Vesicle size distribution profile \& microscopy}

All the batches of flurbiprofen loaded liposomes were examined for their morphological attributes using binocular compound microscope (optics) at suitable magnification ${ }^{21}$. The batches containing non dispersed lipid film, drug precipitate or aggregates were detected and discarded. Scanning electron microscopic analysis was carried out on selected formulations for their morphology 22 .

\section{Determination of drug encapsulation \%}

The percentage drug entrapped was determined by centrifugation ${ }^{24,25}$. Liposomal suspension of $10 \mathrm{~mL}$ was placed in centrifugal tube and it was balanced on the other side with equivalent weight. The centrifugation was carried out at $4500 \mathrm{rpm}$ for $10 \mathrm{~min}$. The supernatant was removed and concentration of the drug in supernatant was determined spectrophotometrically at $247 \mathrm{~nm}$. The pellet obtained was lysed in ethanol, diluted suitably and it was analyzed for the drug concentration. Drug encapsulation \% (DE \%) was determined by following equation, DE\% $=$ Amount of drug in pellet total drug *100, DE\% was calculated for 3 formulations of each batch and averaged.

\section{Stability studies}

The ability of the vesicles to retain the drug was assessed by storing the liposomal suspension at different temperatures such as freezer temperature $\left(-20{ }^{\circ} \mathrm{C}\right)$, refrigerator temperature $\left(4-8{ }^{\circ} \mathrm{C}\right)$, room temperature $\left(25 \pm 2{ }^{\circ} \mathrm{C}\right)$ and $37 \pm 2{ }^{\circ} \mathrm{C}$ for one month ${ }^{26,27}$. Samples were withdrawn periodically and drug content analysis was carried out as mentioned in the determination of drug encapsulation\%. 


\section{In vitro release studies}

Drug release studies were carried out using modified USP XXI dissolution rate model which consists of a $250 \mathrm{~mL}$ beaker and an open ended plastic tube of $17.5 \mathrm{~mm}$ diameter. At one end of the tube sigma membrane was tied and the other end left free. This setup was slightly immersed in the beaker containing $90 \mathrm{~mL}$ of phosphate buffer saline, which was maintained at $37 \pm 2{ }^{\circ} \mathrm{C}$. Liposomic suspension of $10 \mathrm{~mL}$ was placed in the tube. The dissolution medium was stirred magnetically at $100 \mathrm{rpm}$. Dissolution sample of $1 \mathrm{~mL}$ was withdrawn periodically for $24 \mathrm{~h}$ and drug content was analyzed.

\section{Results and Discussion}

\section{Optimization of process parameters}

As a preliminary study, the formulation technique was optimized by studying various process parameters such as rotational speed of rotary evaporator, vacuum pressure, medium of hydration, time of hydration. It was found that thickness and uniformity of the lipid film varies depending upon the rotational speed of the evaporating flask. The optimum speed was found to be $150 \mathrm{rpm}$. The film obtained after rotary evaporation was kept overnight under vacuum to dry \& remove the solvent residuals if any and to avoid the formation of emulsion, which may form due to the presence of residual solvents in lipidic film during hydration. It was found that vortexing did not influence the DE\%, which was confirmed by calculating the DE\% before and after doing vortexing. In addition, 2 min vortexing was found to be optimum to obtain the homogeneous liposomal vesicles. Further it was observed that liposomes prepared using phosphate buffer $\mathrm{pH} 7.4$ as the hydration medium showed better DE\% compared to those prepared with water as the hydration medium.

\section{Preparation of liposomes}

Several batches of liposomes were prepared to study the effect of drug-lipid ratio, effect of alkyl chain length of lipids and effect of cholesterol. First and foremost, four batches containing various drug/SPC mole ratios such as $1: 6,1: 4,1: 3$ and 1:2 were prepared to find out the influence of the same on DE\%. It was found that drug/SPC ratio of 1:3 has given greater drug encapsulation\% compared to others. Drug encapsulation\% was increasing with increasing amount of drug up to (drug/SPC) mole ratio of $1: 3$, followed by a sudden decrease in the DE \% of the drug while attempted to increase DE\% by increasing quantity of the drug in (drug/SPC) mole ratio of 1:2 (Table 1). Thus, DE\% was found to be dependent on the drug/lipid ratio of the formulation.

Secondly, in order to find out the influence of cholesterol which is a common component of liposomes providing rigidity to the membrane, controlling permeability and improving plasma stability in a dose dependent manner ${ }^{29}$, further 3 formulations were prepared using different SPC/cholesterol mole ratio (Table 2). SPC/cholesterol mole ratio of 4:1 was observed to be optimum since it increased the DE\% from $43.82 \pm 0.4 \%$ (formulation with zero cholesterol) to $51.54 \pm 0.9 \%$. This may be due to the fact that incorporation of cholesterol increases the hydrophobicity in the membrane bilayer which may favor the encapsulation of hydrophobic drugs ${ }^{30}$. But it was found, in addition that increasing amount of cholesterol to 2:1 and further to 1:1 (SPC/cholesterol) mole ratio decreases the DE\%, despite, 1:1 (SPC/ cholesterol) mole ratio generally used as the starting ratio for conventional liposomes (Table 2). This might be due to the fact that flurbiprofen molecules and cholesterol prefer to align themselves in the hydrophobic region of the membrane bilayer but there is limited space available for both. So they might compete for this space, resulting in lower DE\% with increasing amount of cholesterol. 
Table 1. Effect of Drug/Lipid ratio

\begin{tabular}{cccc}
\hline Liposome Formulation & $\begin{array}{c}\text { DE\% } \\
\pm \text { SD }\end{array}$ & \%Drug Release & $\begin{array}{c}\text { Vesicle size, } \\
\text { nm } \pm \text { SD }\end{array}$ \\
\cline { 1 - 3 } Drug/SPC (Mole ratio) & $39.41 \pm 0.3$ & 65.4 & $147 \pm 0.5$ \\
1:6 (B1) & $42.83 \pm 0.2$ & 69.3 & $149 \pm 0.6$ \\
$1: 4$ (B2) & $43.82 \pm 0.4$ & 68.6 & $149 \pm 0.8$ \\
$1: 3$ (B3) & $29.51 \pm 0.2$ & 69.3 & $146 \pm 0.6$ \\
\hline
\end{tabular}

Abbreviations used: DE\%-Drug encapsulation \%, SPC-Soy phosphatidyl choline. The results are mean $\pm S D$ of 3 formulations

Table 2. Physicochemical characterization of various liposomal batches: Influence of cholesterol (B5-B7), Longer alkyl chain lipids (B8\&B9), Effect of cholesterol in presence of the stealth moiety (B10\&B11)

\begin{tabular}{lccc}
\hline $\begin{array}{l}\text { Liposome Formulation } \\
\text { (Mole ratio) }\end{array}$ & DE\% & \% Drug Release & Vesicle Size, $\mu \mathrm{m}$ \\
\hline SPC/Cholesterol 4:1 (B5) & $51.54 \pm 0.9$ & 49.6 & $168 \pm 0.78$ \\
SPC/Cholesterol 2:1 (B6) & $39.13 \pm 0.2$ & 48.7 & $173 \pm 0.9$ \\
SPC/Cholesterol 1:1 (B7) & $33.32 \pm 0.8$ & 44.4 & $174 \pm 0.3$ \\
DPPC/ Cholesterol 4:1 (B8) & $59.44 \pm 0.6$ & 43.0 & $178 \pm 0.5$ \\
DSPC/ Cholesterol 4:1 (B9) & $62.31 \pm 0.5$ & 36.6 & $182 \pm 0.36$ \\
DSPC/ Cholesterol/ PE-PEG & $68.32 \pm 0.8$ & 29.1 & $192 \pm 0.62$ \\
4:1:0.2 (B10) & & & \\
DSPC/ Cholesterol/ PE-PEG & $47.23 \pm 0.4$ & 25.4 & $198 \pm 0.6$ \\
2:1:0.2(B11) & & & \\
\hline
\end{tabular}

Abbreviations used: DE\%- Drug encapsulation \%, SPC-Soy phosphatidyl choline, DPPC-Di palmitoyl phosphatidyl choline, DSPC- Distearoyl phosphatidyl choline, PE-PEG- PE 18:0/18:0-PEG 2000. The results are mean $\pm S D$ of 3 formulations

As the third part of our study, two batches of liposomes were prepared containing 2 different lipids with varying alkyl chain length such as DPPC (16 alkyl chain length) and DSPC (18 alkyl chain length) which were compared with SPC (natural phospholipids containing 3 alkyl chain length). These two formulations were prepared with DPPC/cholesterol- 4:1 \& DSPC/cholesterol, - 4:1 mole ratios respectively. Our results indicate that lipids with increasing alkyl chain length increases drug encapsulation \% in the following order, DSPC > DPPC > SPC (Table 2). This might be due to the fact that incorporation of longer alkyl chain lipids increases the hydrophobic area in the lipid bilayer membrane.

The final phase of our study focused on preparation of long circulating liposomes commonly known as stealth liposomes using PE 18:0/18:0-PEG 2000 (PE-PEG) with the mole ratios of DSPC:CH:PE-PEG- 4:1:0.2 and DSPC:CH:PE-PEG-2:1:0.2.The former one was found to have greater drug entrapment than the liposomes prepared with only DSPC/cholesterol. In view of the observations obtained, further increasing the amount of cholesterol to 2:1:0.2 (DSPC:CH:PE-PEG) mole ratio lead to dramatic decrease in drug encapsulation \% and stability. (Table 2).

\section{Fourier transform, infrared (FTIR) study}

Drug, excipients interaction was studied before developing the formulation by using FTIRspectroscopy, which is one of the most important analysis to describe about the stability of 
formulation, presence of drug \& drug release (Figure 1). FTIR spectrum of physical mixture of excipients shows minor shifting of some peaks compared with FTIR spectrum of individual excipients and pure drug like $\mathrm{O}-\mathrm{H}$ stretching of aliphatic acid from 3422.91 to 3426.74, C-H stretching of aliphatic methyl group from 2918.34 to 2918.43, C=O stretching of acid from 1740.48 to $1740.61, \mathrm{C}-\mathrm{H}$ bending of methyl group from 1468.42 to 1468.52, C-O stretching of acid from 1236.17 to 1235.05 . These minor shifts observed may be due to the formation of hydrogen bonds, vanderwaals attractive forces or dipole moment which are weak forces seen in the polar functional groups of drugs and excipients. The shifts seen due to the above mentioned interaction may however support the formation of favorable vesicle shape, structure with good stability and sustained drug release.

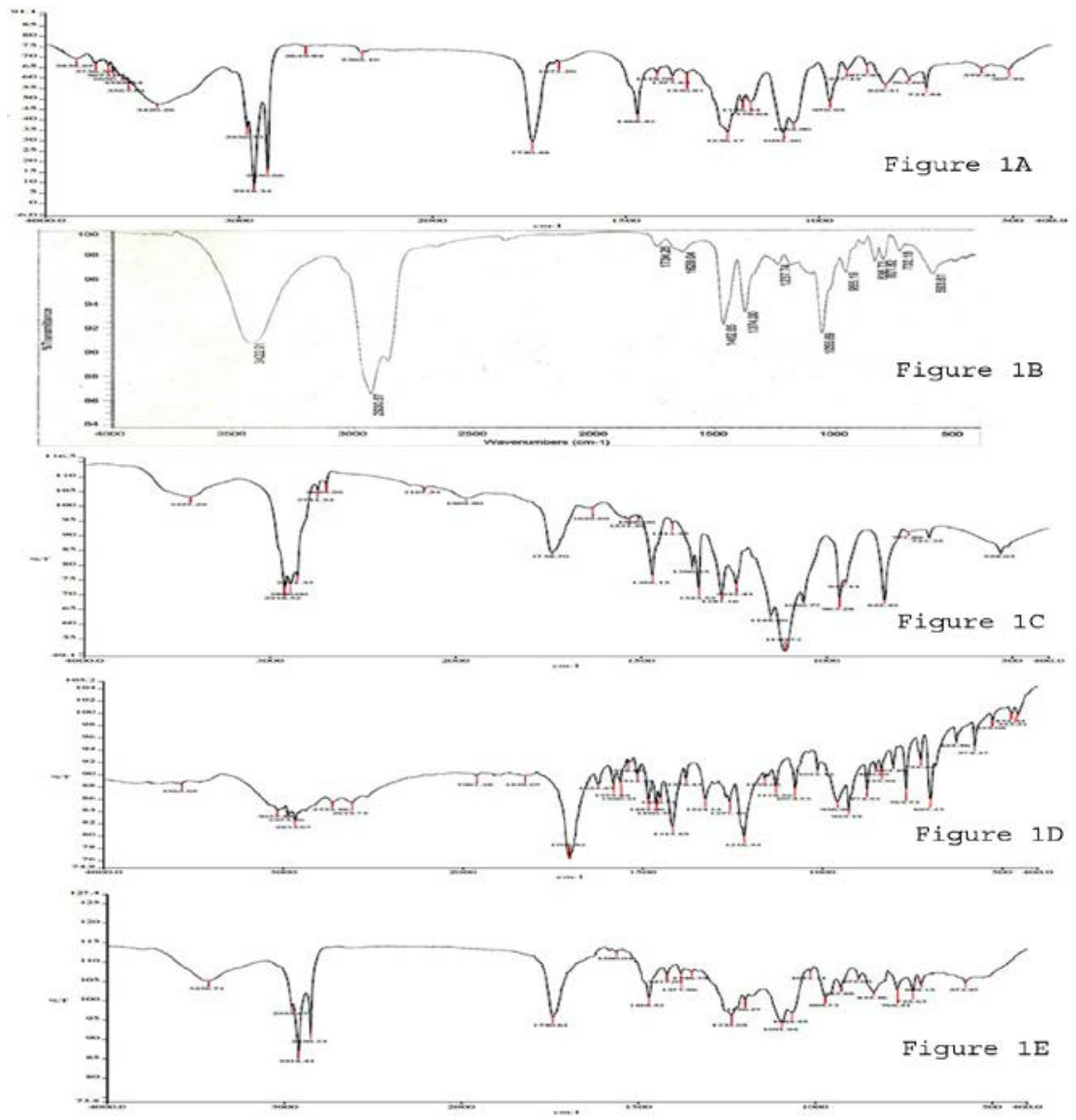

Figure 1. A) FTIR spectrum of DSPC, B) FTIR spectrum of cholesterol, C) FTIR spectrum of PE 18:0/18:0-PEG, D) FTIR spectrum of flurbiprofen, E) FTIR spectrum of physical mixture of flurbiprofen and excipients.

\section{Size distribution profile}

The vesicle size was found to be varied from $146 \pm 0.6 \mathrm{~nm}$ to $198 \pm 0.6 \mathrm{~nm}$. The liposomes were photographed using scanning electron microscope (Figure 2). Vesicles were found to be spherical in shape. Analysis of vesicle size was carried out for 3 formulations of each formulation code and the size was found to be reproducible. 


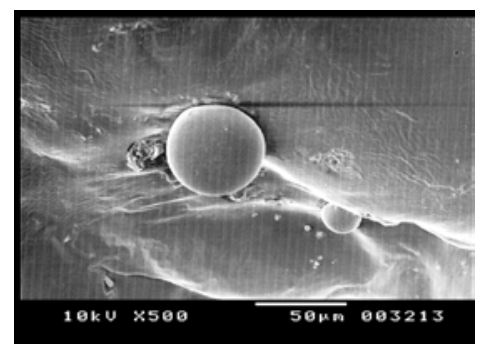

Figure 2. Scanning electron microscope (SEM) picture of liposomal formulation B10

\section{Stability profile}

The selected PEGylated liposome was evaluated for physical and chemical stability by storing the liposomal formulation for 1 month at 4 different temperatures as previously described. There are no significant changes in drug encapsulation\% for the formulations stored at $-20^{\circ} \mathrm{C}$ and $4{ }^{\circ} \mathrm{C}$. But it was found that there was a considerable reduction in $\mathrm{DE} \%$ of the liposomes stored at room temperature as well as $37 \pm 2{ }^{\circ} \mathrm{C}$ (Figure 3 ).

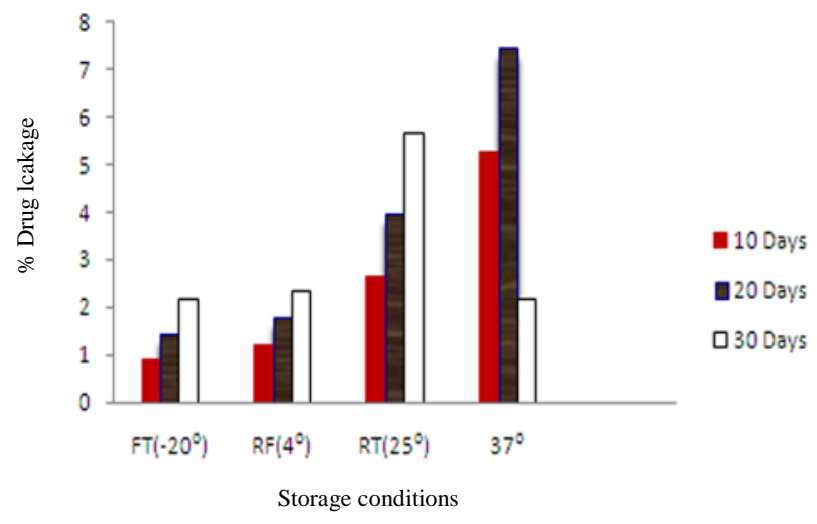

Figure 3. Extent of drug leakage from B10 liposomes at different storage temperatures

\section{In-vitro drug release study}

Among the four formulations prepared without cholesterol to find out the influence of drug/lipid ratio in our study, SPC/drug mole ratio of 4:1 without cholesterol could release the highest amount of the drug in 24 h i.e. $69.3 \%$ followed by SPC/drug mole ratios of 6:1, 3:1 and 2:1 (Table 1), (Figure 8). The drug release behavior of liposomes prepared incorporating cholesterol (B5, B6 and B7 in Table 2) showed improved drug retention capacity and therefore drug release \% was decreased as follows: $49.6 \%, 48.7 \%$ and $44.4 \%$ for the B5, B6 \& B7 respectively (Figure 9). When the percentages of drug were normalized to lipid content of each sample, SPC only liposomes were seen to retain remarkably lower, where as other liposomes could retain drug at higher \%, supporting the fact that highest extent of drug release occurred in cholesterol free liposomes. The in vitro release study was also correlated with retention value of liposomes prepared with long alkyl chain lipids DPPC, DSPC (Table 2). The drug release pattern was found to be in the following order DSPC $<$ DPPC $<$ SPC (Figure 4 and 5). This may be due to the fact that increase in alkyl chain lengh of lipids increases the phase transition temperature, due to stronger vanderwaals interaction between the lipid chains. Thus, higher energy is required to disrupt the ordered 
packing $^{31}$. On the other hand, great lipidic area of longer alkyl chain lipids may enhance the drug binding with lipid bilayer resulting slower or sustained drug release. When compared to the release profile of other liposome formulations, the stealth liposomes prolonged further the drug release. This slower release in stealth liposomes could be because of the fast hydration process of PEG presence on the surface of the vesicles.

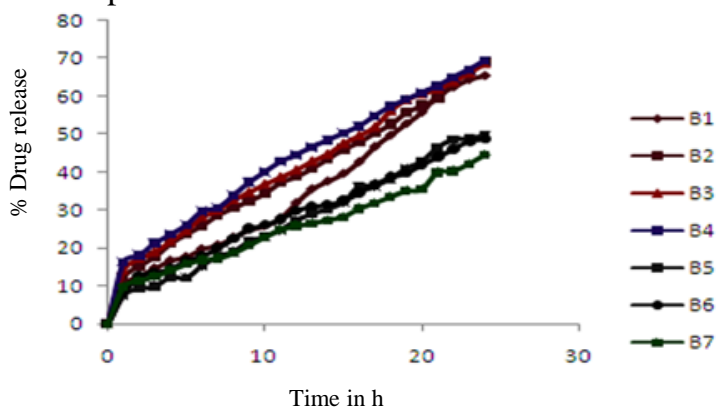

Figure 4. Plots of in vitro cumulative percentage drug release vs. time for different flurbiprofen liposomes of B1-B7

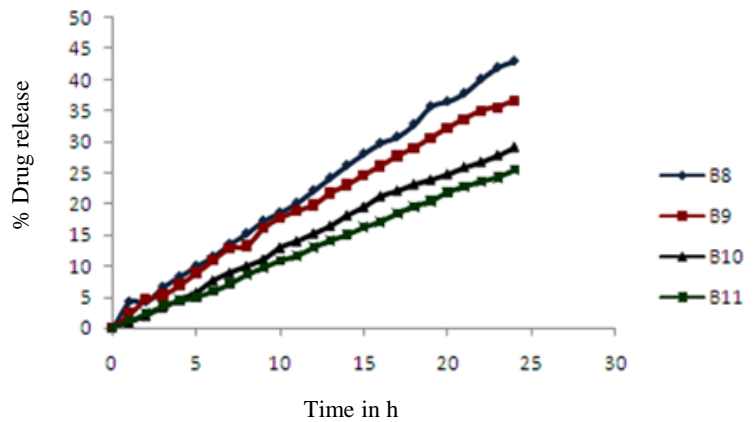

Figure 5. Plots of in vitro cumulative percentage drug release vs. time for different flurbiprofen liposomes of B8-B11

\section{Conclusion}

In the present study, highly hydrophobic drug flurbiprofen was successfully encapsulated in to liposomes composed of different phospholipids with desired characteristics. Flurbiprofen liposomes were shown to be influenced by bilayer cholesterol content and alkyl chain length of lipids. Presence of optimum amount of cholesterol was found to enhance encapsulation efficiency and increasing amount of cholesterol was found to decrease the encapsulation as well as drug release. So the present study has given us knowledge too that liposomes with low amount of cholesterol are better candidates for liposomes of flurbiprofen. This study also confirmed that flurbiprofen retention can be enhanced by employing long alkyl chain length phosphatidylcholines. As an exclusive attempt, flurbiprofen loaded stealth liposomes were also prepared successfully with superior drug loading and drug retention characteristics than the conventional as well as liposomes with long alkyl chain lipids.

\section{References}

1. $\quad$ Alberto A G, Clin Can Res., 2011, 7, 223-225.

2. Danilo D L, Sci Med., 1996, 34-43. 
3. Immordino M L, Dosio F and Cattel L, Int J Nanomedicine, 2006, 1(3), 297- 315.

4. $\quad$ Allen T M, Adv Drug Del Rev., 1988, 2, 55-67.

5. Mauk M R and Gamble R E, Proc Nati Acad Sci USA, 1979, 76, 765-769.

6. Abra R M and Hunt C, Biochim Biophys Acta, 1981, 666, 493-503.

7. Kunie V, Maeda F, Harashima H and Kiwada H, J Pharm Pharmacol., 1990, 43, 162-166.

8. Allen T M and Hansen C, Biochim Biophys Acta, 1991, 1068, 133-141.

9. Gregoriadis G and Senior J, FEBS Lett., 1980, 119(1), 43-6.

10. Allen T M and Cleland L G, Biochim Biophys Acta, 1980, 597, 410-426.

11. Scherphof G L, Damen J and Wilschut J, In: Gregoriadis G, Ed., L.iposome Technology. Boca Raton. FL: CRC Press, 1984, 3, 205-224.

12. Allen T M and Chonn A, FEBS Lett., 1987, 223, 42-46.

13. Gabizon A and Papahadjopoulos D, Proc Nati Acad Sci USA, 1988, 85, 6949-6953.

14. Allen T M, Hansen C and Rutledge J, Biochim Biophys Acta, 1989, 981, 27-35.

15. Allen T M, Ryan J L and Papahadjopoulos D, Biochim Biophys Acta, 1985, 818, 205-210.

16. Chi S C, Park E S and Kim K, Int J Pharm., 1995, 126, 267-274.

17. Charoo N A, Shamsher A A, Kohli K, Pillai K and Rahman Z, Colloids and surfaces B: Biointerfaces, 2008, 65, 300-307.

18. Fang J Y, Hwang T L and Leu Y L, Int J Pharm., 2003, 250, 313-325.

19. Teixeira A V, Pocas L and Serrao P, Br J Clin Prac., 1984, 32, 222-227.

20. Cardoe N, Daymond T J, Risdall P C and Glass R C, Curr Med Res Opin., 1975, 3(S4), 15-19.

21. Kumar R and Katare O P, J Pharm Pharmaceut Sci., 2004, 7, 252-259.

22. Ramana M V, Chaudhari A D, Himaja M, Satyanarayana D and Dua K, Indian $J$ Pharm Sci., 2007, 69, 390-393.

23. Buddhadev L and Biswajit M, Sci Pharm., 2010, 76, 507-515.

24. $\quad$ Pai R S and Devi K V, Int J Pharm Sci Nanotechnol., 2009, 1(4), 317-326.

25. Hathout R M, Mansour S, Mortada N D and Guinedi A S, AAPS Pharm Sci Tech., 2007, 8(1), E1-E12.

26. Manosroi A, Kongkaneramit L and Manosroi J, Int J Pharm., 2004, 270, 279-286.

27. Goril E, Flatten G E, Bunjes H, Luthman K and Brandl M, Eur J Pharma Sci., 2006, 28, 336-43.

28. Rathode S and Deshpande S G, Indian J Pharm Sci., 2010, 72(2), 155-160.

29. Deniz A, Sade A, Keskin D, Tezcaner A and Sreeparna B, Biosci Rep., 2010, 30, 365-373.

30. Subczynski W K, Wisniewska A, Yin J J, Hyde J S and Kusumi A, Biochem., 1994, 33, 7670-7681.

31. Mohammed A R, Weston N, Coombes A G A, Fitzgerald M and Perrie Y, Int J Pharm., 2004, 285, 23-34. 